\title{
Embossing of light trapping patterns in sol-gel coatings for thin film silicon solar cells
}

\author{
Maurits C.R. Heijna ${ }^{*}$, Jochen Löffler ${ }^{\mathrm{a}}$, Bas B. Van Aken ${ }^{\mathrm{a}}$, Wim J. Soppe ${ }^{\mathrm{a}}$, \\ Rob van Erven ${ }^{\mathrm{b}}$, Ronald Franken ${ }^{\mathrm{b}}$, Herman Borg ${ }^{\mathrm{b}}$, Patrick P.G.J.M. Peeters ${ }^{\mathrm{b}}$ \\ ${ }^{a}$ ECN Solar Energy, Westerduinweg 3, 1755 LE, Petten, the Netherlands; \\ ${ }^{\mathrm{b}}$ OM\&T B.V., Glaslaan 2, 5616 LW, Eindhoven, the Netherlands
}

\begin{abstract}
For thin-film silicon solar cells, light trapping schemes are of uppermost importance to harvest as much as possible of the available sunlight. Typically, one uses randomly textured front TCOs to scatter the light diffusively in pin-cells on glass. Here, we investigate methods to texture the back contact with both random and periodic textures for use in nip-cells on opaque foil. We applied an electrically insulating SiOx-polymer coating on a stainless steel substrate, and textured this barrier layer by embossing. On this barrier layer the back contact is deposited for further use in the solar cell stack. Replication of stamps with various random and periodic patterns was investigated, and, using scanning electron microscopy, replicas were found to compare well with the originals. Masters with U-grooves of various submicrometer widths have been used to investigate the optimum dimensions of regular patterns for light trapping in the silicon layers. Angular reflection distributions were measured to evaluate the light scattering properties of both periodic and random patterns. Diffraction gratings show promising results in scattering the light to specific angles, enhancing the total internal reflection in the solar cell.
\end{abstract}

Keywords: thin film solar cells, light trapping, nanoimprint

\section{INTRODUCTION}

Thin film microcrystalline silicon solar cells are an emerging PV technology as a cheap alternative to the more traditional wafer-based crystalline silicon solar cells since they can be produced using low-cost manufacturing methods [1]. However, the thickness of the absorber layer in these cells, $1 \mu \mathrm{m}$ versus $200 \mu \mathrm{m}$ for wafers, is too thin for the light to be sufficiently absorbed in one pass. As a result, thin film microcrystalline Si solar cells typically need light trapping schemes to increase the pathway and to thus enhance the absorption [2], especially for the red and near infrared part of the spectrum [3].

Light scattering in thin film silicon solar cells is usually achieved by nanotextured back reflectors and/or nanotextured transparent conducting oxide (TCO) front electrodes. For $p-i-n$ "superstrate" devices, glass with randomly textured front TCO electrodes is widely used; the textured TCO is typically made by APCVD, MOCVD or by sputtering and texture etching [3-5]. An alternative to the use of random structures is the use of periodic structures like diffraction gratings to trap light in solar cells. These gratings are quite appealing in that they diffract light into the solar cell under specific angles, which may be larger than the angle of total internal reflection, thus trapping the light inside the cell. Regular patterns are produced in TCO front layers by the elaborate technique of photolithography [6, 7].

In the ECN cell concept for roll-to-roll production of thin film Si solar cells, $n-i-p$ cells are deposited on an opaque flexible substrate consisting of steel foil covered with an electrically insulating barrier layer and a back contact and back reflector layer (see Figure 1). The use of a metal foil allows for deposition at optimum temperatures in the range of $200^{\circ} \mathrm{C}$, but requires an electrically insulating barrier layer to electrically separate the substrate from the back contact which is essential to allow monolithic series interconnection [8]. In this paper we demonstrate that by embossing the insulating barrier layer this layer can induce light scattering, thus adding extra functionality to this layer. Morphological

*heijna@ecn.nl; phone +31 224 564953; www.ecn.nl 
and optical characterization of various original and replicated random and periodic light scattering structures is presented to demonstrate the versatility of this method to produce substrates for thin film silicon solar cells.

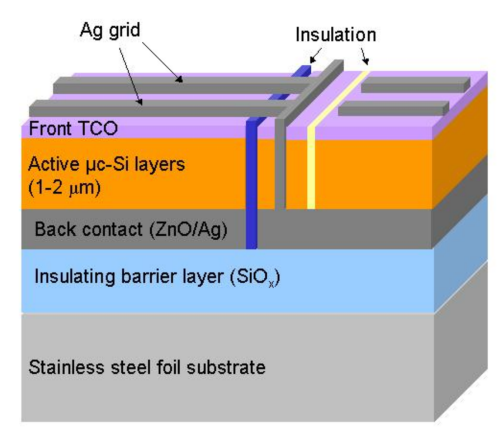

Fig. 1. Schematic representation of the ECN cell concept for thin film silicon solar cells. A flexible metal substrate is used in combination with an insulating barrier layer to allow for monolithic series interconnection. Note that for clarity, the layer thickness and lateral dimensions of the series interconnection are not drawn to scale. (Color online)

\section{EXPERIMENTAL}

\subsection{Embossing procedure}

The insulating layer consists of a thermally curing $\mathrm{SiO}_{\mathrm{x}}$-polymer sol-gel coating that can by applied by either spray coating or roll coating. After application, the layer is dried for a short period of time to such an extent that the coating is dust-dry but still is plastically deformable. At this point, a texture can be applied to the sol-gel layer by embossing. A master structure with a maximum surface area of 10 by $10 \mathrm{~cm}^{2}$ is placed on top of the coated steel foil with its texture towards the coating. The texture is transferred by applying pressure in a hydraulic press with heated anvils, resulting in the inverse of the original morphology being stamped into the coating. Several periodic and random master structures were tested for their replication and optical properties. Due to the dust-dry surface of the sol-gel layer, an anti-sticking layer is not required on these masters. Metal masters with diffraction gratings were used for the 1-D periodic structures. These gratings have a groove depth of $300 \mathrm{~nm}$, and periods of 500, 750, and $1000 \mathrm{~nm}$. To create substrates with randomly rough textures, Asahi U-type $\left(\mathrm{SnO}_{2}: \mathrm{F} \mathrm{TCO}\right)$ and "hot silver" were used. Asahi U-type glass is commercially available, and can be used without any further surface treatment. 'Hot' silver masters can be made by sputtering of silver on glass using high $\left(\sim 400^{\circ} \mathrm{C}\right)$ substrate temperatures. The high substrate temperature results in a rough surface with lateral features in the range of one to several microns, depending on sputtering conditions.

\subsection{Characterization of masters and replicas}

To compare the surface morphology of masters and their replicas, scanning electron microscopy (SEM) and atomic force microscopy (AFM) was used. The optical properties of the masters and replicas were compared by measuring the haze parameter and the angular distribution function (ADF). The replicas were coated with a $40 \mathrm{~nm}$ aluminum layer for the optical measurements, because the layer itself is transparent.

The wavelength-dependent haze parameter $\mathrm{Hz}$ is defined as the ratio between the diffusely reflected light intensity and the total reflected light intensity, and is given by

$$
H z(\lambda)=\frac{R_{\text {diffuse }}(\lambda)}{R_{\text {total }}(\lambda)}
$$

with $\lambda$ the wavelength, $R_{\text {diffuse }}(\lambda)$ the wavelength-dependent diffuse reflection, and $R_{\text {total }}(\lambda)$ the wavelength-dependent total reflection. The total reflection of a surface is measured using a spectrometer in combination with an integrating sphere to capture all reflected light. The diffuse reflection is measured with the same set-up but the specular reflection is cancelled out by a light trap placed in the integrating sphere. For amorphous silicon the wavelength range relevant for light trapping is $600-800 \mathrm{~nm}$, whereas for microcrystalline this range extends from 600 to $1000 \mathrm{~nm}$ [3]. 
The haze parameter does not contain information on the angular distribution function of the diffusely reflected light. For light trapping, high angles of reflection are beneficial as these increase the percentage of light internally reflected at the $p$-Si/front-TCO and TCO / encapsulant (or air) interface. For angles of reflection larger than the angle of total internal reflection, all light will be reflected back into the absorber layer. One can calculate the angle of total internal reflection $\theta_{\text {tir }}$ by using

$$
\theta_{t i r}=\arcsin \frac{n_{T C O}}{n_{S i}}
$$

Assuming a refractive index at $600-800 \mathrm{~nm}$ for amorphous $\mathrm{Si}$ of $n_{\mathrm{a}-\mathrm{S}} \approx 3.5$, and for TCO of $n_{\mathrm{TCO}} \approx 2$, the $p$-Si/TCO interface has $\theta_{t i r} \approx 35^{\circ}$, which is taken as an indicative angle for evaluating light scattering substrates.

To measure the ADF of the reflected light of masters and replicas, an optical Goniometer (Instrument Systems GON360) was used in combination with a halogen light source and an Avantes diode array optical spectrometer in the range of 400-1000 nm. The light source and the detector are connected to the goniometer by optical fibers. The signal-to-noise ratio of the scattered light from random textures is too low to use the white light source. The ADF of these samples was measured at $632 \mathrm{~nm}$ using a He-Ne laser instead of the halogen lamp.

The main function of the barrier layer is to electrically separate the back contact from the metal substrate. The insulation behavior should be retained after texturization of the layer. In order to verify the insulating properties, resistance and break-down voltage were measured on both flat and textured layers at voltages of 100 up to $1000 \mathrm{~V}$ on surface areas of $4 \times 4 \mathrm{~mm}^{2}$ and $10 \times 10 \mathrm{~mm}^{2}$.

\section{RESULTS AND DISCUSSION}

\subsection{Random textures}

Replicas were made in the insulating layer using a sample of Asahi U-type $\mathrm{SnO}_{2}$ :F-coated glass as master. Figures $2 \mathrm{a}$ and $b$ show SEM images comparing the surface morphology of the Asahi master with its replica. The replica of the Asahi U-type texture shows an inverted and somewhat rounded off structure with respect to its original. The SEM images of the hot Ag original and replica show significant difference in the typical length scale of the morphology (figure $2 \mathrm{c}$ and $\mathrm{d}$ ). The original has large grains in the order of $0.5-3 \mu \mathrm{m}$ with grooves at the grain boundaries. Upon replication these grooves become protruding features on top of the surface with typical dimensions of 100-500 nm. The grains replicate as larger flat areas in between these protrusions.

For the optical reflection measurements, both the original Asahi U-type glass and the sol-gel replica were coated with a $40 \mathrm{~nm}$ aluminum reflective layer by e-beam evaporation. Although the Asahi structure in the replica is inverted with respect to the original, the haze parameter is comparable, showing only little difference (Figure 3 ).

Also shown in figure 3 is the haze parameter for hot silver master and replica textures. For these textures the haze parameter of the replica deviates significantly from that of its original. Especially at longer wavelengths, in the range of 600 to $1000 \mathrm{~nm}$ that is relevant for microcrystalline silicon, the haze parameter of the replica is typically 0.2 (absolute value) lower than the original. As a result of the change in surface morphology, the light scattering properties at longer wavelengths have decreased upon replication.

The angular distribution at $632 \mathrm{~nm}$ shows a steeper angular dependence of the scattered light for the hot-silver samples than for the Asahi-U samples (figure3b). The scattered intensity at $632 \mathrm{~nm}$ is lower at all angles for the Asahi-U type than for the hot Ag, in accordance with the haze data at this wavelength. Although the haze for the hot Ag replica is quite high, the largest part of the light is scattered into angles smaller than $\sim 40^{\circ}$. In a solar cell, this rear reflector will lead to increased light absorption, but total internal reflection will only be obtained for a small fraction of the incident light. 


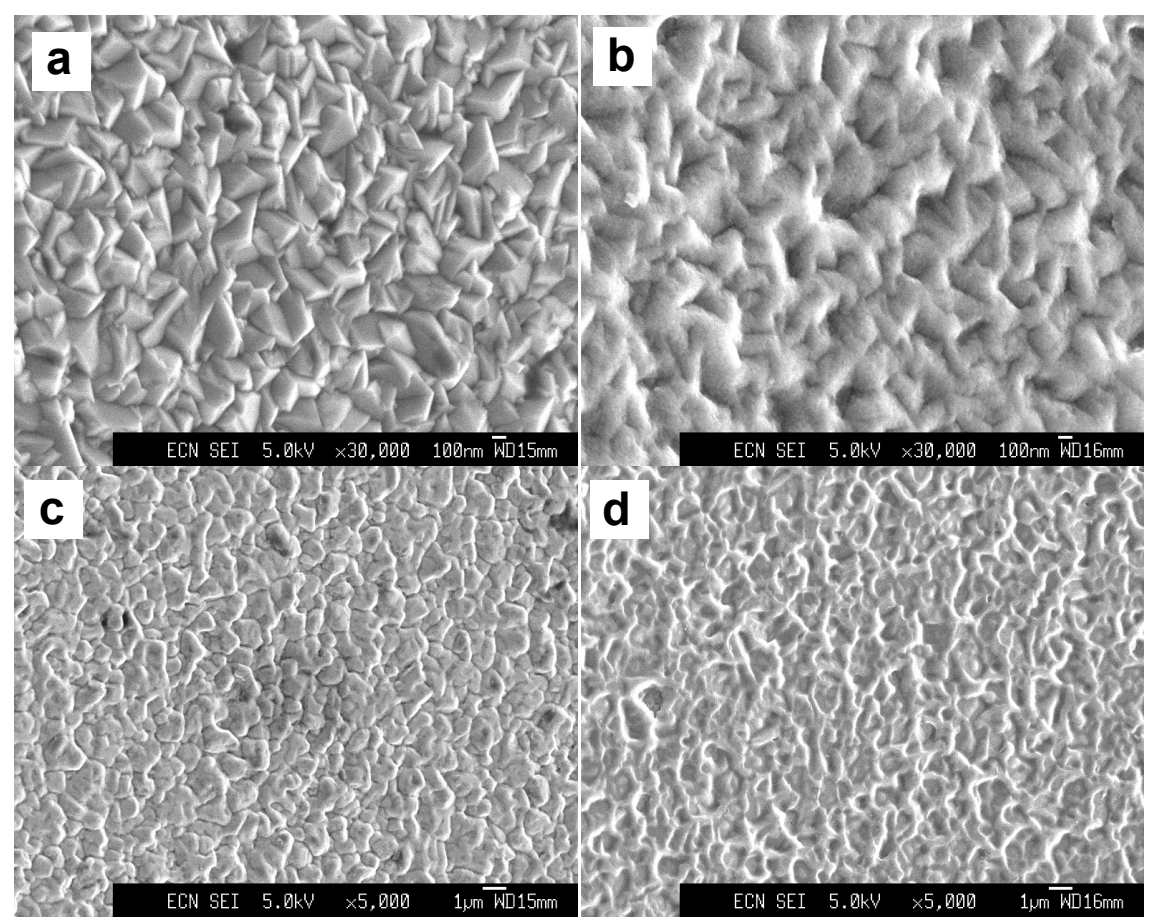

Fig 2. SEM images of randomly textured masters an their replicas in the barrier layer. (a) Asahi U-type SnO:F-coated glass. (b) Replica in the barrier layer. The texture of the replica is inverted with respect to that of the original, and has a $40 \mathrm{~nm}$ Al layer deposited on top of it for optical reflection measurements. (c) Sputtered "Hot Ag" on glass. (d) Replica of the hot Ag structure in the barrier layer.
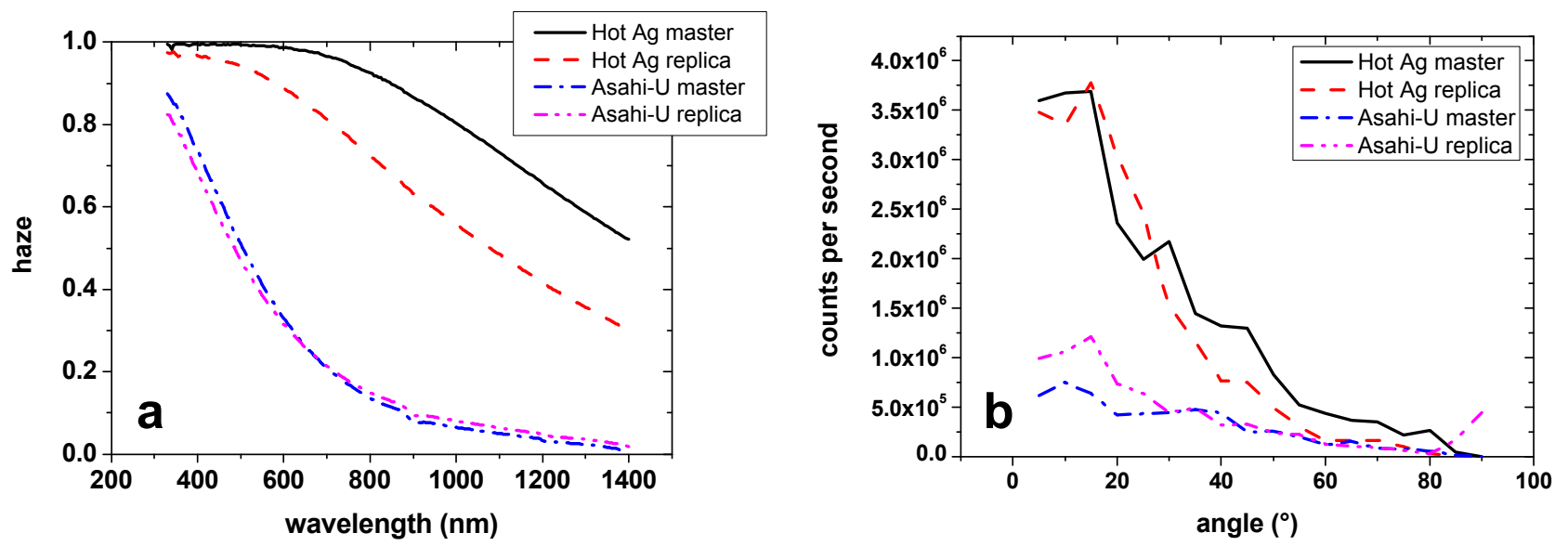

Fig 3. (a) Haze of randomly textured masters and replicas. (b) Angular distribution function of reflected light at a wavelength of $632 \mathrm{~nm}$, for randomly textured masters and replicas.

\subsection{Periodic textures}

Figure 4 shows SEM images of a metal master with a one-dimensional diffraction grating and its replica. This and other tested gratings comprise U-shaped grooves of $300 \mathrm{~nm}$ deep, with periods of 500, 750, and $1000 \mathrm{~nm}$. For all three periods, the replicas compare well with their originals. Figure 5 shows height profiles taken from AFM measurements on the $1000 \mathrm{~nm}$ period master and its replica. Upon replication, the grating looses approximately $50 \mathrm{~nm}$ in height with respect to the $300 \mathrm{~nm}$ high original. The shape is somewhat rounded as a result of material of the replication layer not being able 
to reach the corners of the master grooves. Note that the walls of the grooves cannot be imaged properly due to tip convolution effects.
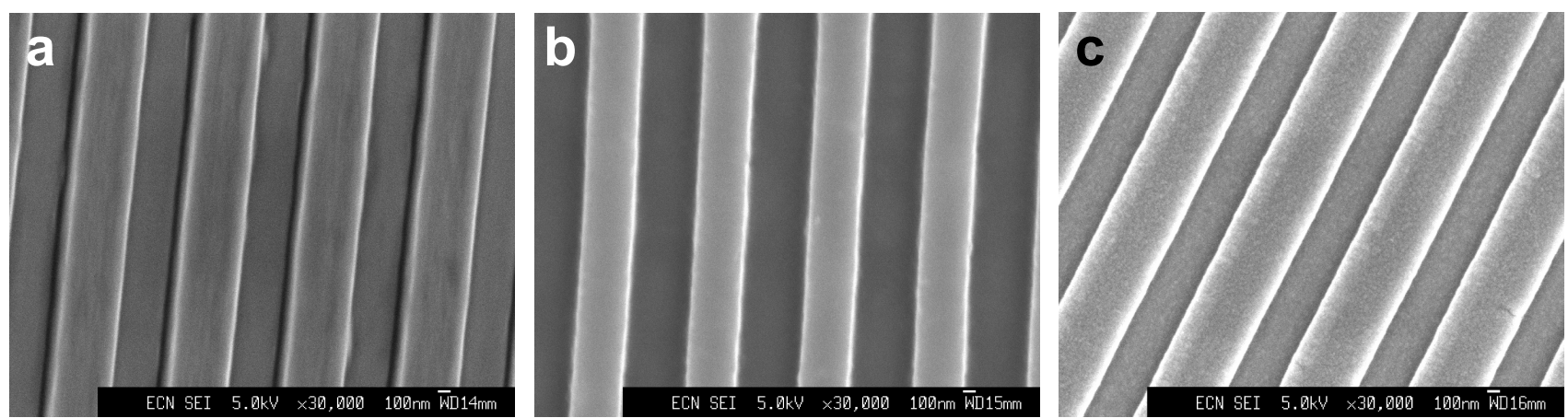

Fig 4. SEM images of (a) a metal master with a $1000 \mathrm{~nm}$ period grating, (b) its replica in the sol-gel insulating layer, and (c) its replica in the insulating layer, covered with a silver and zinc oxide back contact layer. The scale bar in all three images represents $100 \mathrm{~nm}$.

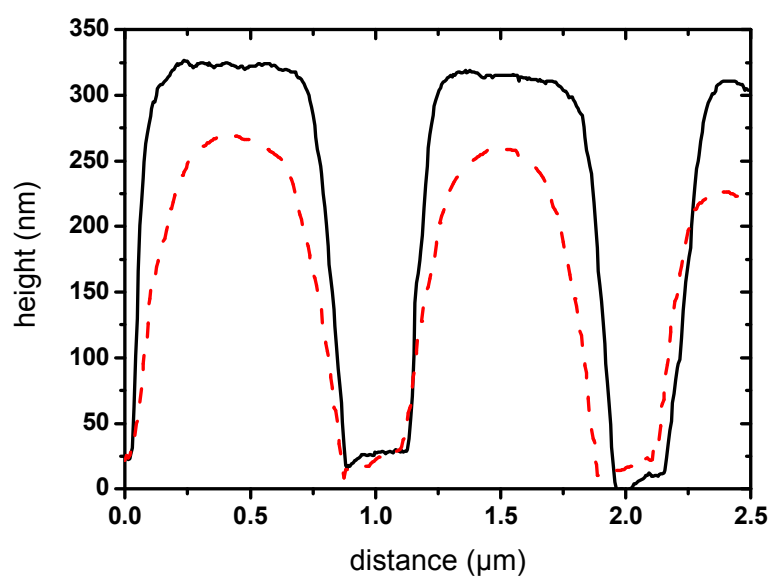

Fig 5. Height profiles taken from AFM images of a $1000 \mathrm{~nm}$ period master grating (solid line), and its replica in the sol-gel insulating layer (dashed line).

Haze measurements on the masters and Al-covered replicas show that for wavelengths larger than the period of the grating no diffraction occurs (figure 6). At wavelengths equal to the period of the grating, the angle of diffraction $\theta$ is $90^{\circ}$, following the diffraction formula given by

$$
\sin \theta=m \frac{\lambda}{p}+\sin \theta_{i}
$$

with $\lambda$ the wavelength, $m$ the diffraction order, $p$ the period of the grating, and $\theta_{i}$ the angle of incidence. For higher wavelengths no diffraction occurs and the reflection is specular. Qualitatively the haze of the masters and Al-covered replicas match, but quantitatively there are deviations due to the difference in dielectric function. 

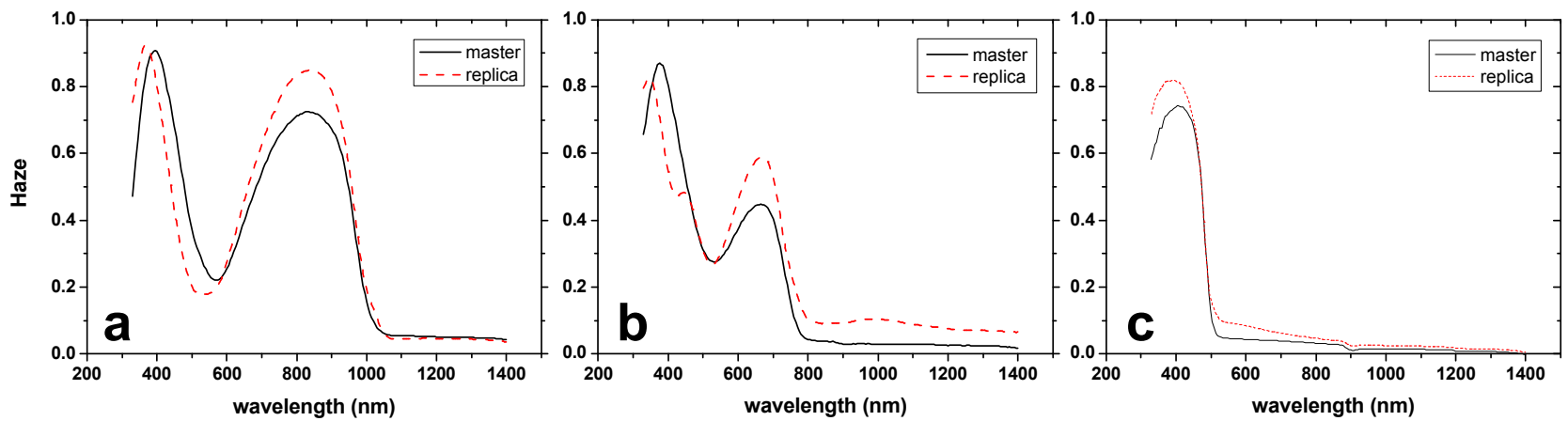

Fig 6. Haze measurements on masters and replicas with diffraction gratings of (a) $1000 \mathrm{~nm}$ period, (b) $750 \mathrm{~nm}$ period, and (c) $500 \mathrm{~nm}$ period

Figure 7 shows the ADF of the reflected light for replicated diffraction gratings relative to the intensity of the incident light. As can be expected, the wavelength-dependent angle of diffraction follows the theoretical relation of equation (3) for all three replicas. For the $1000 \mathrm{~nm}$ period also the second order diffraction $(\mathrm{m}=2)$ is visible, for the other two gratings the second order diffraction peaks fall outside the wavelength range of the detector and source. For both the $750 \mathrm{~nm}$ and $1000 \mathrm{~nm}$ grating the lower intensity of diffracted light around $500 \mathrm{~nm}$ leads to reduced haze around this wavelength. The diffracted intensities of the gratings correspond well to the haze measurements. For instance, at wavelengths around 800 $\mathrm{nm}$ the ADF shows the diffraction peaks to have approximately $40 \%$ of the incident light intensity. A similar ADF is present for negative angles, thus a total of $80 \%$ of the incident light is diffracted at an angle of $\pm 60^{\circ}$ at a wavelength of $800 \mathrm{~nm}$, in correspondence with the haze at this wavelength (dashed line in figure 6a). For the estimated angle of total internal reflection of $35^{\circ}$, the diffraction equation (eq. 3) shows that the grating with $1000 \mathrm{~nm}$ period has the optimal diffraction for use with amorphous silicon and at longer wavelengths for microcrystalline silicon. The experimental ADF shows this grating to have the highest diffraction intensity of the gratings tested in this paper. For amorphous silicon, the grating with $750 \mathrm{~nm}$ period may also be used as its ADF approximately matches the relevant wavelength range of 600$800 \mathrm{~nm}$, but has significantly lower diffracted intensity than the $1000 \mathrm{~nm}$ period in this range. The $500 \mathrm{~nm}$ grating however is not suited for use as rear side reflector as above $500 \mathrm{~nm}$ all light is reflected specularly.
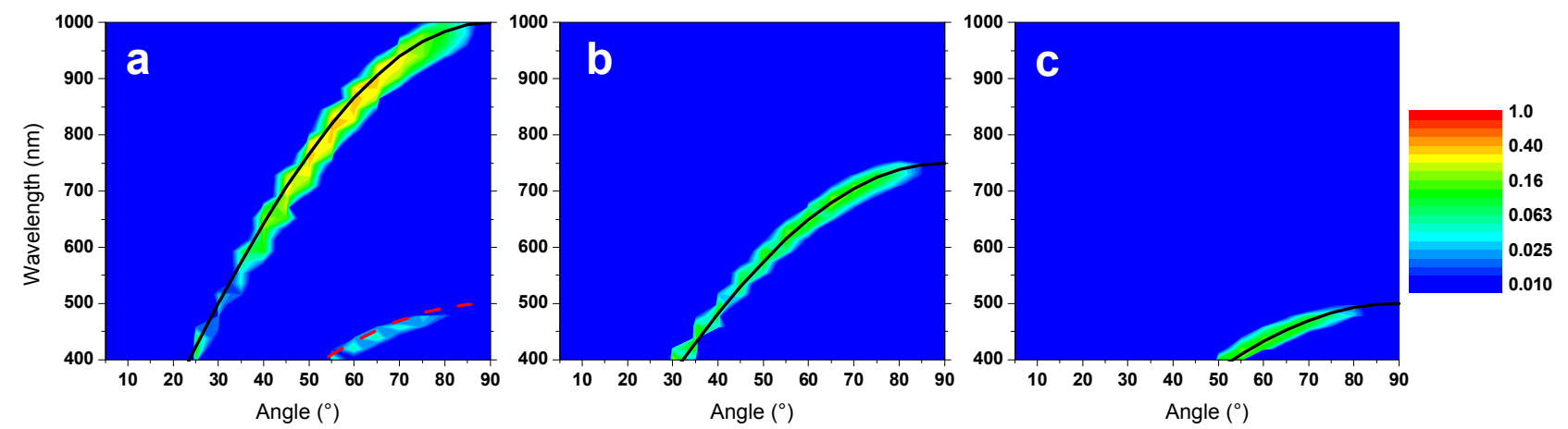

Fig 7. Angular distribution functions for replicated diffraction gratings at perpendicular incident light. The horizontal axis indicates angle of reflection, the vertical axis the wavelength, and the colors the intensity of the reflected light relative to the incident light $(=1)$ on a logarithmic scale. (a) $1000 \mathrm{~nm}$ period grating, with the theoretical relation between angle and wavelength indicated by the solid (first order) and dashed (second order) lines. (b) $750 \mathrm{~nm}$ period. (c) $500 \mathrm{~nm}$ period (color online).

\subsection{Plasmonic absorption in back contacts}

Textured back contacts can increase the short circuit current by scattering the light in the absorber layer of a solar cell. A possible drawback is that rough surfaces and gratings may give rise to enhanced absorption of light due to surface plasmon resonances [9] and antireflective properties due to an effective refractive index gradient (effective medium 
theory). Figure 8 shows total reflection measurements on flat and textured surfaces covered with $80 \mathrm{~nm}$ silver and $60 \mathrm{~nm}$ $\mathrm{ZnO}$. The flat reference surface, also covered with $\mathrm{Ag} / \mathrm{ZnO}$, is part of the same sample to cancel out any effects due to deposition inconsistencies. The flat part of the samples have a low roughness, which results in a small plasmonic absorption peak at around $400 \mathrm{~nm}$ [10]. The Asahi-U replica shows a broad absorption peak around $600 \mathrm{~nm}$ of up to $25 \%$ loss which is related to the typical length scale of the small inverted pyramids on its surface (Figure 2b). For the periodic structures (Figure 4c shows the $1000 \mathrm{~nm}$ period grating with $\mathrm{Ag} / \mathrm{ZnO}$ layer), the reflection spectra show multiple absorption dips, related to the period of the respective gratings. The absorption peaks exclude losses due to index gradients as in the effective medium theory. A balance needs to be found between the gain in light trapping and the absorption loss increased by textured back contacts. The resonant wavelengths depend on surface structure and dielectric constant of the $\mathrm{ZnO}[9,10]$, giving a means to shift the plasmonic resonances to outside the wavelength range of interest, e.g increasing the dielectric constant of the $\mathrm{ZnO}$ to shift the resonance to longer wavelengths.
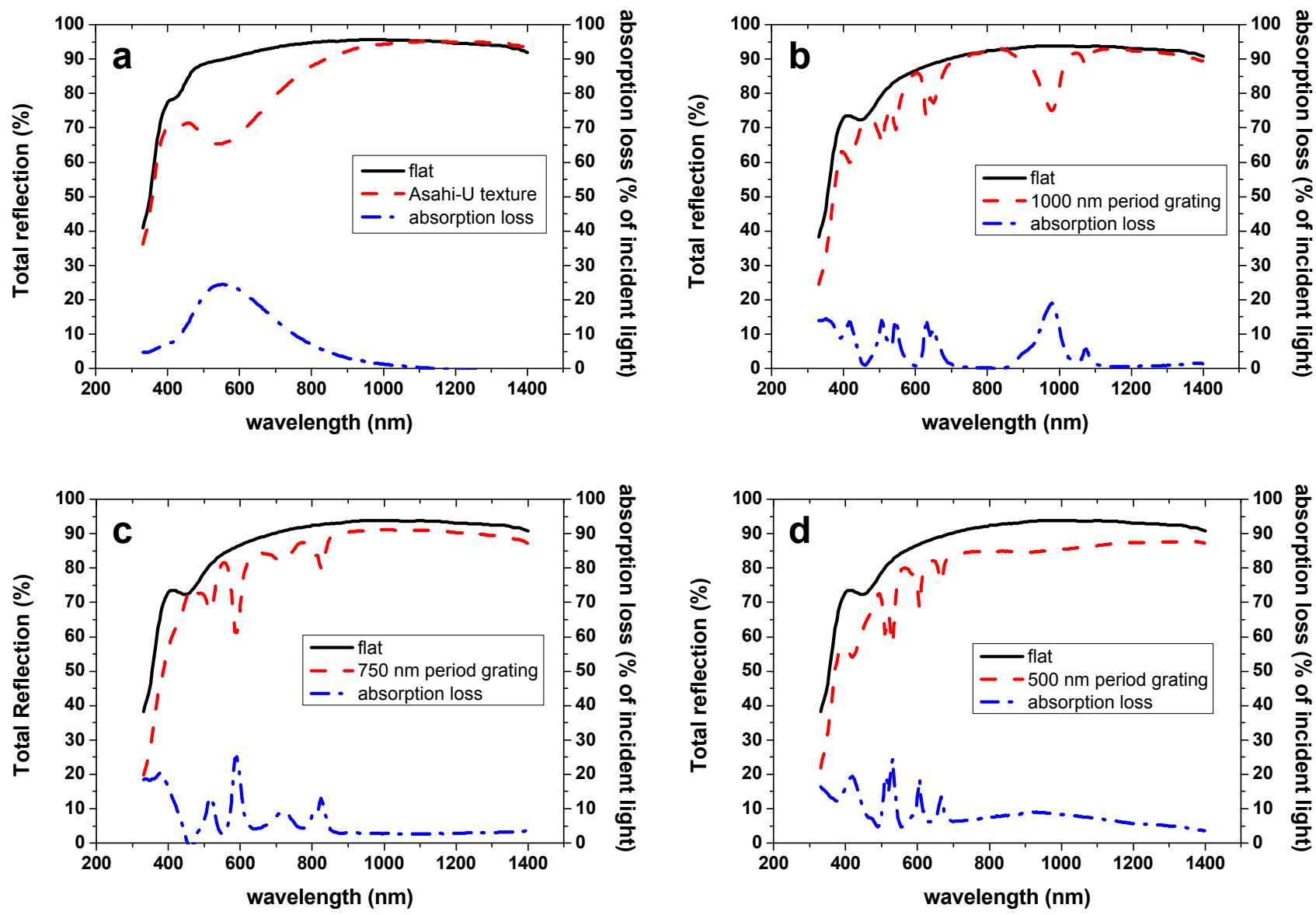

Fig 8. Spectral total reflection measurements on flat and textured $\mathrm{Ag} / \mathrm{ZnO}$ surfaces. (a) Reflection spectrum of Asahi U-type replica covered with $\mathrm{Ag} / \mathrm{ZnO}$ compared to the spectrum of a flat section of the same sample. The dash-dotted line indicates the difference between flat and textured reflection, attributed to plasmonic losses. Measurements on replicated gratings show a clear relation between period and absorption losses; (b) $1000 \mathrm{~nm}$ period, (c) $750 \mathrm{~nm}$ period, (d) $500 \mathrm{~nm}$ period.

\subsection{Insulating properties}

A periodic structure consisting of a mixture of submicron grooves was used to test the electrical insulation of textured barrier layers with various feature sizes in one experiment. The flat reference sample was part of the same $10 \mathrm{by}^{2} \mathrm{~cm}^{2}$ piece of coated foil, in order to cancel out any differences in layer properties between barrier layer depositions. Table 1 shows the average breakdown voltage and resistance value. Although texturing the foil reduces the resistance of the layer typically by a factor 4 , the breakdown voltage remains higher than $1000 \mathrm{~V}$. Thus, the insulating properties remain well within the specifications for application in the ECN solar cell concept. 
Table 1. Resistances R100V and R500V measured at 100 and $500 \mathrm{~V}$ DC, respectively, and breakdown voltage of flat and textured barrier layers for $0.16 \mathrm{~cm}^{2}$ and $1 \mathrm{~cm}^{2}$ contact pads.

\begin{tabular}{|c|c|c|c|}
\hline & $\begin{array}{l}R_{100 \mathrm{~V}} \\
(\mathrm{G} \Omega)\end{array}$ & $\begin{array}{l}R_{500 \mathrm{~V}} \\
(\mathrm{G} \Omega)\end{array}$ & $\begin{array}{c}\text { V }_{\text {breakdown }} \\
\text { (V) }\end{array}$ \\
\hline Flat, $0.16 \mathrm{~cm}^{2}$ & 165 & 93 & $>1000$ \\
\hline Flat, $1 \mathrm{~cm}^{2}$ & 89 & 33 & $>1000$ \\
\hline Textured, $0.16 \mathrm{~cm}^{2}$ & 40 & 20 & $>1000$ \\
\hline Textured, $1 \mathrm{~cm}^{2}$ & 10 & 4 & $>1000$ \\
\hline Required, $0.16 \mathrm{~cm}^{2}$ & & $6-30$ & $>1000$ \\
\hline$\overline{\text { Required, } 1 \mathrm{~cm}^{2}}$ & & $1-5$ & $>1000$ \\
\hline
\end{tabular}

\section{CONCLUSION}

Light trapping structures are of high importance for thin-film microcrystalline silicon solar cells. We have demonstrated that by means of embossing random and periodic textures can be replicated in sol-gel barrier layers without loss of the insulating functionality of these layers. The angular distribution of reflected light suggest that periodic grating patterns with a period of 750 to $1000 \mathrm{~nm}$ are suited to enhance the light trapping in thin film Si solar cells. Embossing of sol-gel layers offers a versatile method to replicate light-trapping structures of any choice for solar cell substrate production. An optimization step of the texture and back reflector composition is needed to reduce the effect of (plasmonic) absorption in the back contact.

\section{ACKNOWLEDGEMENTS}

This work has been financially supported by the Dutch Ministry of Economic Affairs (Project No. EOSLT04029). AFM measurements have been performed at FOTON, INSA, Rennes.

\section{REFERENCES}

[1] Maycock, P. and Bradford, T., "PV Technology, Performance, and Cost, 2007 Update", Prometheus Institute for Sustainable Development and PV Energy Systems, 2007.

[2] Deckman, H. W., Roxlo, C. B. and Yablonovich, E., "Maximum statistical increase of optical absorption in textured semiconductor films", Opt. Lett. 8, 491-493 (1983).

[3] Müller, J., Rech, B., Springer, J. and Vanecek, M., "TCO and light trapping in silicon thin film solar cells", Solar Energy 77, 917-930 (2004).

[4] Meier, J., Kroll, U., Vallat-Sauvain, E., Spitznagel, J., Graf, U., and Shah, A., "Amorphous solar cells, the micromorph concept and the role of VHF-GD deposition technique", Solar Energy 77, 983-993 (2004)

[5] Sato, K., Gotoh, Y., Wakayama, Y., Hayashi, Y. Adachi, K., and Nishimura, H., Reports of the Research Labs, Asahi Glass Co., Ltd. 42, 129 (1992).

[6] Senoussaoui, N., Krausse, M., Müller, J., Bunte, E., Brammer, T. And Stiebig, H., "Thin-film solar cells with periodic grating coupler", Thin Solid Films 451-452, 397-401 (2004)

[7] Eisele, C., Nebel, C.E. and Stutzmann, M., "Periodic light coupler gratings in amorphous thin film solar cells“, J. Appl. Phys. 89, 7722-7726 (2001)

[8] Löffler, J., Ballif, C., Brecl, K., Brooks, K., Finck, C., Fischer, D., Haug, F.-J., Mayerhofer, R., Soppe, W.J., Späth, M., Topic, M. and Wutz, M., Technical Digest of PVSEC 17, Fukuoka, Japan, (2007)

[9] Raether, H., [Surface plasmons on smooth and rough surfaces and on gratings], 1988, Springer Verlag. 
SPIE Optics \& Photonics 2008 - San Diego; 7047-4

[10] Springer, J., Poruba, A., Mullerova, L., Vanecek, M., Kluth, O., and Rech, B., “Absorption loss at nanorough silver back reflector of thin-film silicon solar cells”, J. Appl. Phys. 95, 1427-1429 (2004) 